\title{
El vínculo de pareja: Una posibilidad afectiva para crecer
}

\author{
Couple's Bond: An Emotional Possibility to Grow
}

\author{
Juan Ortega Rojas ${ }^{1}$ \\ División de Educación para el trabajo \\ Centro de Investigación y Docencia en Educación \\ Universidad Nacional \\ Heredia, Costa Rica \\ jortega1@costarricense.cr
}

Recibido 15 de marzo de 2012 - Corregido 22 de octubre de 2012 • Aceptado 07 de noviembre de 2012

Resumen. El vínculo de pareja se constituye en uno de los más importantes que construyen las personas en sus vidas. Tal relevancia implica, por un lado, la revisión de esquemas personales, sobre el concepto que se ha creado sobre este y, por otro, la disposición de aprender cosas nuevas $y$, con estas, asumir actitudes que tienen que ver con varios aspectos. Dentro de estos, podrían mencionarse los siguientes: i) aceptar a la persona tal cual es, en tanto algunos rasgos no atenten contra la dignidad de la otra, ii) mantener apertura constante a la comunicación, en particular, en momentos de crisis, iii) conocerse y valorarse a sí mismos, antes de conocer y valorar al otro, iv) mantener o crear detalles que, de antemano, se sabe producen gratificación en la pareja, v) respetar compromisos adquiridos y vi) tratar de vivenciar cosas, a través del movimiento de roles como una de las estrategias. Cada uno de esos elementos, debidamente desarrollados, se constituye, a su vez, en recomendaciones que, de ser adecuadamente implementadas, facilitan la creación de vínculos más saludables y duraderos. Desde este marco, el artículo pretende fomentar la revisión de ideas y conductas propias, en relación con el vínculo de pareja. Invertir tiempo en desarrollar actitudes y conductas pareciera ser el camino que debe seguirse, para la construcción de un vínculo de pareja placentero, dentro en un ambiente de crecimiento mutuo.

Palabras claves. Pareja, vínculo, aceptación, comunicación, compromiso, respeto, detalles, cambio, roles, historia personal.

\footnotetext{
1 Magíster en Psicología Clínica Grupal, Licenciado en Ciencias de la Educación con énfasis en Orientación. Orientador del Ministerio de Educación Pública, Asesor Regional de Orientación de este mismo Ministerio. Actualmente académico de la Universidad Nacional y orientador de la Universidad Estatal a Distancia.
} 
URL: http://www.una.ac.cr/educare

\begin{abstract}
The bond between a couple is one of the most important ties developed in people's life. Its significance implies examining our personal beliefs on that concept and being open to learn and adopt certain attitudes on different aspects in the relationship, such as: i) accepting the other person the way he or she is, as long as his/her character do not damage the other person's dignity, ii) constant communication, especially in times of crisis, iii) understanding and valuing oneself to understand and value the other person, iv) being thoughtful to please the other person, v) complying with commitments made and vi) trying new experiences by changing roles as one of the strategies. These aspects become recommendations which appropriately undertaken will facilitate the creation of healthier and longer lasting bonds. In this context, this paper aims at encouraging the examination of your own behavior and ideas, in terms of couple's bond. Taking some time to develop different attitudes seems to be the way to achieve a pleasant bond within an environment of mutual growth.
\end{abstract}

Keywords. Couple, bond, acceptance, communication, commitment, respect, details, change, roles, personal story.

Como seres sociales que somos, los seres humanos, en su mayoría, mantenemos, a lo largo del ciclo vital, diversidad de vínculos, dentro de los cuales y según algunos teóricos (Buscaglia, 1994; Satir, 1988; Villarreal, 1997), el de pareja ocupa un lugar muy importante. Sin embargo, y aun con tal relevancia, es común encontrar personas que manifiestan sentirse frustradas o desilusionadas como producto de vínculos o del rompimiento de algunos que parecían buenos.

Antes de continuar, sería importante distinguir entre vínculo y relación. En este sentido, decir que el vínculo de pareja es algo más que una relación tiene que ver con lo expuesto por Lerner (2003) cuando afirma que el primero implica lazos afectivos importantes, una historia en común, un espacio donde la comunicación y el compromiso están presentes. Por el contrario, en la relación la inversión de energía afectiva es menor.

De acuerdo con lo anterior, es de suponerse que la relación de pareja vendría a ser un vínculo por lo que este implica y no una mera relación. En este sentido, el vínculo implicaría cierta armonía dentro de un proceso mutuo de dar y recibir.

Por lo anterior, resulta de suma importancia, ante la necesidad personal de un vínculo de pareja, tener claro el concepto que se maneja de lo que es un vínculo de esta naturaleza, en términos de las necesidades que se pretenden llenar con él y, a la vez, lo que se tiene para dar.

En este sentido es común escuchar a personas decir: "Mis relaciones no funcionan porque mi pareja no me llena". Esta afirmación la encontramos usualmente en hombres y mujeres que a partir de su historia de vida llegan con muy poco que ofrecer esperando que su pareja llene totalmente dicho vacío.

Es comprensible lo anterior, si entendemos como causal de tal situación que en dicha historia de vida hubo quizá carencias afectivas que en el aquí y ahora impiden la construcción 
de un vinculo saludable. Sobre este particular, teóricos como Buscaglia (1998) y Lerner (2003) plantean que el amor como una de las bases para la creación del vinculo con uno mismo y con los otros tiene sus raíces en experiencias de crianza tempranas. Sin embargo, no por esta razón las personas no podrán edificar relaciones y vínculos saludables que implicarían, desde lo cognitivo, entender que si bien es cierto la pareja contribuye, efectivamente, en la satisfacción de una o más necesidades, dicho aspecto desde lo conductual se esperaría que fuese mutuo. No obstante y para no tener como consecuencia inmediata el fracaso, se hace necesario hacer revisiones y cambios en los esquemas mentales de los cuales se profundizará mas adelante.

Dentro de este marco y dando al vinculo de pareja un lugar preponderante dentro del desarrollo de la persona, es que a continuación se desarrollan algunos factores que desde la experiencia, y desde lo que mencionan los autores y las autoras son importantes en su construcción.

\section{Aceptar a la persona tal cual es}

Esta recomendación, aplicable a cualquier tipo de relación y vínculo, es de gran importancia, pues la mayoría de las personas aspiran a que las otras actúen según su esquema de referencia previo, llámese a esto las estructuras creadas en etapas tempranas de la vida. A este proceso de pretender que los otros y otras sean y actúen bajo los esquemas propios, es lo que Bernard (1988) Ilama sobreposición del mundo interno sobre el mundo externo.

Aprender a discriminar lo mío de los demás es indispensable en el vínculo de pareja, pues de esta forma se permite al otro u otra actuar según las características de su personalidad. No obstante, esta aceptación del otro u otra en términos de fortalezas y debilidades no aplica en aquellos rasgos que atenten contra la integridad de una de las dos personas.

Es importante entender que la persona que se elija como pareja es un conglomerado de rasgos y como tal va a poseer una serie de características consideradas como deseables y otras que quizá no lo sean.

\section{Mantener líneas de comunicación abiertas}

Se ha dicho que la comunicación es la base del éxito en la interacción con las personas y, en efecto, esto pareciera ser muy pertinente. Pero en la cotidianidad y en momentos de crisis, particularmente, no es tan fácil como parece, pues existe la tendencia a repetir patrones de comunicación según lo aprendido en vínculos primarios y en relaciones previas.

Como punto inicial, ante el interés de crear y consolidar vínculos de pareja, sería importante recordar el aporte de Ospina (1983), Satir (1998) y Villarreal (1997) sobre los patrones de comunicación construidos con personas con quienes se tuvo relaciones o vínculos en etapas previas, en particular dentro del núcleo familiar. Es muy probable que de estas personas se 
URL: http://www.una.ac.cr/educare

haya aprendido la forma de comunicarse en la actualidad con los otros y otras, a través de modelos saludables o asertivos o bien agresivos o disfuncionales. De ahí la sugerencia que plantean los mismos autores de autorevisarse en cuanto a estas herencias comunicacionales.

Una vez realizada esta revisión, se estaría en capacidad de hacer modificaciones o bien mantener la forma de comunicarse que se ha utilizado hasta el momento, si se considera que ha sido saludable en términos de asertividad, respeto, afecto, etc.

En todo caso, abrir canales de comunicación entre la pareja vendría a significar que ambas personas asuman el compromiso de compartir lo que corresponde a un vínculo, es decir, desacuerdos, logros, metas en común, necesidades, etc., o bien desarrollar la disposición de aprender a hacerlo.

Este aprendizaje puede resultar difícil porque implica modificar estructuras de pensamiento que, con el paso del tiempo, se han reforzado y manifestado de formas que se pueden catalogar como nocivas para la comunicación saludable, tales como silencios, agresiones, rupturas de acuerdos y otras que atenten con la llamada comunicación asertiva, definida por Satir (1988) como la capacidad de expresarse de una forma directa, honesta y respetuosa.

\section{Consolidar el vínculo a partir de autovinculaciones: el papel de la autovaloración}

La autoestima, definida como el amor por uno mismo, tiene, según Mézerville (2004), dos fuentes, una interna que es precisamente ese amor de la persona por sí misma, producto de una autoevaluación positiva en términos de un reconocimiento claro de fortalezas y limitaciones; y por otro lado, una fuente externa relacionada con lo proveniente de los otros. En este sentido, las palabras y gestos -sean estos positivos o negativos- vendrían a afectar el valor que la persona se atribuye a sí misma.

De esta segunda fuente externa forma parte la persona que se eligió para compartir dentro del vínculo de pareja.

Un compartir que se vuelve más provechoso y placentero cuando se sabe que ese otro y otra es capaz de reconocer y expresar opiniones positivas en torno a sí mismo, a su pareja y al vínculo como tal, considerando como requisito para hacer ver al otro y otras sus fortalezas el poder reconocerlas en primera instancia en uno mismo. Es decir, difícilmente se puede ver aspectos positivos en la otra persona, si resulta difícil o imposible verlos en si misma.

Sobre la misma idea y en lo referente a la pareja, esta actitud de reconocer las fortalezas y logros del otro no solo revitaliza el vínculo, sino que también produce el efecto de devolución, en donde el intercambio de palabras y gestos positivos se vuelve parte de la cotidianidad.

Sin embargo, es relevante tener claro que ese intercambio de palabras y gestos positivos que favorecerían el crecimiento de la pareja no implique la fusión de ambos; por el contrario, dicho crecimiento se daría sobre la base de mantener como parte del vínculo: el tú, el yo y el 
nosotros; en este sentido, ninguno perderá la identidad (se mantiene el tú y el yo), pero se construirá un tercer espacio que abarca lo de ambos (el nosotros) compuesto por aquellas experiencias, metas, logros que involucran a los dos. Con estas condiciones se pueden reconocer las fortalezas y logros del otro, tomando en cuenta que es en los espacios individuales del tú y el yo, donde hay oportunidad de mirar al otro u otra desde otro ángulo. El nivel de crecimiento de ese nosotros dependerá entonces del aporte que ambos den al vínculo y de la satisfacción que se sienta en el compartir con ese otro, lo cual a su vez es una consecuencia del disfrute que cada uno tiene del sí mismo como ser individual.

\section{Mantener por siempre los detalles}

Es usual que en la etapa de enamoramiento los seres humanos muestren una serie de actitudes que llevan como fin la conquista. Podemos hablar de palabras, mensajes escritos, obsequios entre otra gran variedad.

Estos detalles, que en un inicio tuvieron como consecuencia favorecer el enamoramiento, con frecuencia se pierden a medida que el vínculo avanza hacía otras etapas. Pareciera ser que una vez cumplido el objetivo de conquista, ya no tiene sentido el mantener aquello que facilitó el proceso.

De acuerdo con Buscaglia $(1994,1998)$ los seres humanos tenemos la valiosa capacidad de enamoramiento y de amar. No obstante, tal estado se mantiene en tanto exista la motivación necesaria para asumir el compromiso que el vínculo de pareja requiere.

Dicha motivación no emerge de la nada. Son esos detalles que a veces parecieran insignificantes los que hacen que ambas personas sientan gratificación y, con ella, el deseo de continuar. Son esas actitudes y conductas las que permiten aun en fuertes crisis consolidar el vínculo, en vez de terminarlo.

¿Cuáles son esos detalles? Esa es la pregunta que cada quien tiene que contestarse. Investigar con detenimiento y amor qué cosas hacen sentir bien a la otra persona y aprovechar fechas que son importantes como aniversarios o cumpleaños para sorprenderla (o) con uno de esos detalles; o bien hacerlo en cualquier momento cuando menos lo espera. Y sobre todo mantener esa actitud por siempre; no importa si se tiene uno o veinte años de unión.

Ahora bien, por historia de vida algunas personas presentan resistencia a dar y recibir afecto a través de las diversas manifestaciones. No obstante, es ahí donde se puede asumir la actitud y la apertura a aprender. El dar y recibir afecto es una conducta que podríamos Ilamar innata; basta con ver a los niños pequeños para darnos cuenta de esto. Son las experiencias a tempranas edades las que pueden hacer que las personas se resistan a amar y a ser amadas. Sin embargo, estoy convencido de que la necesidad de afecto desde el contacto físico y desde las manifestaciones orales son una necesidad humana, y podría ser el vínculo de pareja un espacio para reparar heridas y crecer en el vinculo tal cual se ha descrito. 
URL: http://www.una.ac.cr/educare

\section{Respetar los compromisos adquiridos}

La decisión de construir un vínculo implica, al igual que en todas las decisiones, que se pasará de un estado a otro. En este cambio de estado hay ganancias y hay pérdidas; y si el termino pérdidas no gusta, hablemos de que se dejará de hacer cosas que antes se hacían. En otras palabras se tiene que hacer necesariamente una redistribución de tiempos, sin hacer tampoco grandes renuncias que a la final podrían llevar a la pareja a fusionarse, lo cual es sumamente dañino, en el sentido de que se necesitan, como se mencionó anteriormente, espacios individuales. No obstante, vincularse con otra persona implica estar en disposición de respetar a través del cumplimiento, una serie de compromisos que en algunos casos se pueden ver incluso como cargas. Por eso, reitero, la importancia de analizar profundamente lo que implica construir y mantener un vínculo de pareja. Es importante pensar en los espacios compartidos en términos de tiempo y afecto; estos momentos cortos o largos mantendrán el vínculo y lo fortalecerán a través del tiempo.

Es conveniente y recomendable que la pareja hable sobre estos compromisos y se asuma lo que se tenga que asumir por mutuo acuerdo. Los compromisos pueden ser variados según intereses y circunstancias; a modo de ejemplo la fidelidad al otro u otra es tema que muchas parejas incluyen dentro de sus compromisos.

La idea sobre los compromisos es que si no se está en disposición de asumirlos no es el momento de construir un vínculo. Quizá lo que se busque es una relación pasajera que satisfaga alguna necesidad temporal o una relación de larga duración que no implique el involucramiento que se logra al vincularse con las gratificaciones propias de este proceso.

\section{Moverse de roles y vivenciar cosas nuevas}

Se habló, en uno de los apartados anteriores, de mantener por siempre los detalles y de aprender a darlos y recibirlos en particular en aquellas personas que por su historia de vida no desarrollaron esa actitud y conducta. Parte de estos detalles es tratar, en la medida de lo posible, de vivenciar y experimentar cosas nuevas, en tanto estas nuevas experiencias no atenten contra la dignidad de ninguna de las dos personas.

Podría ser que algunas experiencias nuevas hacía las cuales hay resistencia impliquen el moverse de roles asumidos y de ahí la actitud de rechazo. No obstante, tales movimientos podrían resultar gratificadores una vez que se intenten hacer. En todo caso, si la sensación es desagradable, hay posibilidad de volver atrás e intentar otras.

En este sentido, se trataría de hacer variaciones a aquellas cosas que se ha acostumbrado hacer; lo cual permite el refrescamiento del vínculo y, a la vez, descubrir nuevas formas de disfrute en pareja. 
Se dice en el lenguaje popular que la rutina "mata las relaciones". Esta rutina se podría asociar con lo llamado por Pichón-Riviere (1985) como cristalización de roles, en donde las personas se ubican en un lugar de supuesto "confort" y no se atreven a moverse, aun cuando tales movimientos, desde este mismo autor, fortalecen los vínculos y contribuyen al crecimiento individual $y$, en conjunto, de quienes lo conforman. En particular, en la pareja el hacer cosas nuevas lleva a redescubrirse, y a descubrir en el otro y otra elementos revitalizantes.

De esta forma, el principio de conocimiento de sí mismo (a), del cual nos habla Mézerville (2004) como base para el autoestima, aplica a ambas personas, en términos de conocer día tras día aquellas cosas que facilitan el crecimiento de las dos personas.

\section{Conclusiones}

A partir de lo anterior y a manera de conclusiones, se puede anotar que el vínculo de pareja desde lo que podemos llamar saludable, y en concordancia con lo expuesto anteriormente, es un proceso complejo que requiere de la disposición de ambos para asumir una serie de aptitudes y compromisos que resultan valiosos, si se tiene claridad del porqué es importante vincularse.

Indispensable tomar en cuenta que todas las personas traen consigo al vínculo una historia de vida, y que si por uno u otro motivo se han desarrollado resistencias a dar y recibir afecto, cabe la posibilidad de hacer cambios, partiendo de la idea de que mientras haya vida, las oportunidades de cambio existirán.

Es importante destacar, y a modo de recomendación, que lo desarrollado anteriormente se refiere a actitudes y conductas que se deben dar mutuamente en un ambiente favorable a la comunicación, apertura, amor, etc.

Tomar en cuenta que aprender a amar es un proceso, para algunos (as) quizá no fácil. Sin embargo, algunas experiencias que resultan realmente gratificantes y valiosas, a veces cuestan. Implica cambiar algunas formas de pensar y disposición para moverse de roles asumidos y aprendidos en otros momentos, en otras circunstancias y con otras personas.

En todo caso es importante considerar que existe la alternativa de volver atrás y volver a intentarlo y, si finalmente se decide no vincularse a este nivel, también es válido. En este sentido, no es obligatorio ni un deber tener pareja. Sin embargo, no nos daremos cuenta de esto si no se hace el intento.

\section{VALE LA PENA EL ESFUERZO}




\section{Referencias}

Bernard, M. (1988). Interacción entre grupo interno y grupo externo (Maestría en estudios en Psicología Grupal, clase 9). En UCl, Antología: Desarrollo a través de la cooperación (pp. 1-9). Argentina: Instituto de psicoanálisis de las configuraciones vinculares.

Buscaglia, L. (1994). Como amarnos los unos a los otros. El desafío de las relaciones humanas. México: Diana.

Buscaglia, L. (1998). El amor: La experiencia más importante en la vida. México: Editorial Diana.

De Mézerville, G. (2004). Ejes de salud mental. Los procesos de autoestima, dar y recibir afecto y adaptación al estrés. México: Trillas.

Lerner, L. (2003). Del automaltrato a la autoestima. En busca de la salud. Argentina: Lugar Editorial.

Ospina, H. (1983). La orientación familiar. San José, Costa Rica: Ediciones Promesa.

Pichón-Rivière, E. (1985b). Teoría del vínculo. Bueno Aires: Ediciones Nueva Visión.

Satir, V. (1998). Nuevas relaciones humanas en el núcleo familiar. México: Pax.

Villarreal, C. (1997). (compiladora). La orientación familiar: Hacía una nueva perspectiva de la vida en familia: Memoria de seminario. San José, Costa Rica: Oficina de publicaciones de la Universidad de Costa Rica.

\section{Cómo citar este artículo, según APA:}

Ortega, J. (2012). El vínculo de pareja: Una posibilidad afectiva para crecer Revista Electrónica Educare, 16 (Especial), 23-30. Consultado de http://www.revistas.una.ac.cr/index.php/EDUCARE/issue/view/418

Nota: Para citar este artículo en otros sistemas puede consultar el hipervínculo "Como citar el artículo" en la barra derecha de nuestro sitio web: http://www.revistas.una.ac.cr/index.php/ EDUCARE/index 